\title{
Agora tu: Um instrumento lúdico para o desenvolvimento de competências
}

\section{sócio-emocionais}

\section{Now it's up to you: A playful tool for the development of socio-emotional skills}

\author{
Carlos Marinho, Vera Ramalho \\ Psiquilibrios, Centro de consulta Psicológica
}

\begin{abstract}
Resumen
A presente comunicação tem como objetivo apresentar o jogo "Agora tu" como um instrumento de trabalho psicoterapêutico para crianças dos 7 aos 10 anos. O Jogo é utilizado com periodicidade semanal, durante 10 semanas, com a duração de 50 minutos. Pretende-se com este instrumento: (i) Estimular a identificação dos sentimentos no próprio e nos outros; (ii) Promover a comunicação dos sentimentos verbal e não-verbal; (iii) Promover o respeito pelos sentimentos dos outros; (iv) Promover o conhecimento da expressão de sentimentos; (v) Lidar de forma adequada com os sentimentos negativos; e, (vi) Facilitar tomada de decisão e melhorar as relações interpessoais.

Palavras-chave: Jogo, desenvolvimento sócio-emocional
\end{abstract}

\section{Abstract}

This communication aims to present the game "Agora tu" as a psychotherapeutic work tool for children from 7 to 10 years. The Game is used weekly for 10 weeks, lasting 50 minutes. The aim of this instrument is: (i) To stimulate the identification of feelings in oneself and in others; (Ii) Promote communication of verbal and non-verbal feelings; (Iii) Promote respect for the feelings of others; (Iv) Promote knowledge of the expression of feelings; (V) Handle appropriately with negative feelings; And (vi) Facilitate decision-making and improve interpersonal relationships.

Keywords: Emotion play, socio-emotional development

\section{Competência emocional e competência social:} Clarificação de construtos

Do ponto de vista desenvolvimental, e atendendo às suas funções expressivas e comunicativas, as emoções são reconhecidamente alicerçais para a orientação e motivação do funcionamento adaptativo do indivíduo (Silva \& Freire, 2014), promovendo a resolução das tarefas de desenvolvimento características de cada fase do ciclo vital (Abe \& Izard, 1999). Também na literatura científica se constata inequivocamente a influência exercida pelas emoções em processos intrapessoais, como as respostas fisiológicas, a memória, a atenção, a tomada de decisão, e ainda em processos interpessoais, como as interações sociais (Gross \& Thompson, 2007; Morris et al., 2007; Thompson, 1991, cit. Silva \& Freire, 2014). Com efeito, as interações e relações interpessoais apresentam-se como contextos privilegiados para a manifestação das experiências emocionais, da mesma forma que esses processos interativos são orientados e marcados pelas emoções, de onde a indestrinçável ligação entre o indivíduo e o meio no estudo das experiências emocionais (Denham, 2007; Lazarus, 2006). Na medida em que se tornam responsáveis por transmitir os valores e significados associados ao modo como o ser humano estabelece e mantém as suas relações nos mais diferentes contextos e dimensões sociais, as emoções assumem-se como instrumentos essenciais na concretização do processo de socialização (Lourenço, Palmeira, Dinis \& Gouveia, 2010). Nesta sequência, Dennis, Cole, Zahn-Waxler e Mizuta (2002) salientam a importância da partilha de afetos positivos, da contingência emocional e da sensibilidade dos cuidadores como elementos capazes de promover o sucesso do processo de socialização. É neste contexto que emergem, inseparáveis, as noções de competência emocional (CE), e competência social (CS).

\section{A competência emocional}

Historicamente, o construto de Competência Emocional (CE) surge com Saarni (1999), na sequência do conceito teórico de Inteligência Emocional, primeiramente desenvolvido por Salovey e Mayer (1990). Saarni (1999) define competência emocional como a "demonstração da auto-eficácia em transacções sociais que produzem emoções". Segundo Bohnert, Crnic e Lim (2003), as competências emocionais são constituídas por um conjunto de capacidades dinâmicas, indissociáveis e interdependentes, a saber: a compreensão emocional, a expressão emocional e a regulação emocional, as quais enformam o uso das “emoções para uma vantagem plena" (Gross, 1998, p. 287).

Compreensão emocional. De uma forma geral, a compreensão emocional pode ser definida como traduzindo a capacidade de identificar e reconhecer estados emocionais através de expressões faciais, a capacidade de identificar e/ou antecipar emoções despoletadas por situações sociais comuns, e a capacidade de discernir entre as próprias emoções e as 
emoções dos outros (Bosacki \& Moore, 2004; Denham, 2007). Para além disto, a compreensão emocional traduz-se na utilização adequada de vocabulário emocional, incluindo também um conjunto de competências que permitam aos indivíduos reconhecer a natureza, as causas, as consequências e as possibilidades de controlar e regular as suas emoções (Pons, Harris, \& Rosnay, 2000 cit. Belacchi \& Farina, 2010). Com base numa revisão da literatura acerca das investigações realizadas no âmbito do desenvolvimento da compreensão emocional, Pons, Harris e Rosnay (2004) identificaram nove componentes da CE: (i) reconhecimento da expressão facial de emoções; (ii) compreensão das causas externas das emoções; (iii) compreensão de emoções baseadas em desejos; (iv) compreensão das emoções baseadas em crenças; (v) compreensão da influência de "pistas" emocionais; (vi) compreensão da regulação de uma emoção experienciada; (vii) compreensão da possibilidade de esconder uma emoção; (viii) compreensão de diferentes emoções simultâneas; e (ix) compreensão de emoções morais. Esta componente é impactante ao nível das relações sociais da criança (Kang \& Shaver, 2004).

Expressão emocional. Por seu turno, a expressão emocional é considerada o lado visível e partilhado das emoções (Rubin \& Daniels-Byrness, 1983). Ou seja, consiste na capacidade de expressar e manifestar emoções, as quais (idealmente) devem ser expressas de acordo com os objetivos do indivíduo e, simultaneamente, atendendo à sua adequabilidade ao contexto social em que são manifestadas (Denham, 2007).

Regulação emocional. Eisenberg e Spinrad (2004), definem a regulação emocional como o "processo de iniciar, evitar, inibir, manter ou modular a ocorrência, forma, intensidade ou duração de estados de sentimentos internos, fisiológicos, relacionados com emoções, processos atencionais e/ou concomitantes comportamentais das emoções ao serviço de uma adaptação social ou biológica relacionada com o afecto ou atingir objectivos individuais" (Eisenberg \& Spinrad, 2004, p. 338, cit. Melo, 2005).

A competência social. Del Prette e Del Prette (2005) definem Competência Social pela capacidade de articulação, organização e integração de pensamentos, sentimentos e comportamentos em função de objectivos individuais, da adaptação a diferentes situações e das mais variadas demandas sociais, com o intuito de obter um desempenho positivo para o indivíduo, gerador de consequências positivas nas relações com os outros.

Para Yale e Selman (1989, cit. Martins, 2012), os efeitos positivos ao nível das relações interpessoais do indivíduo (Cia \& Barham, 2009, cit. Martins, 2012; Del Prette \& Del Prette, 2005), implicam uma gestão de emoções, pensamentos e ações atendendo aos objetivos pessoais e à capacidade de se adaptar às diversas situações sociais, ressaltando o papel da regulação emocional como mediador no desenvolvimento dessas competências sociais.

Componentes da competência social. A literatura científica identifica duas componentes determinantes da competência social das crianças: a qualidade do seu comportamento social num dado contexto, e o estatuto social por si adquirido no grupo de pares (Asher, 1990). A primeira compreende subcomponentes como as habilidades sociais eficazes, a ausência de comportamentos inadaptativos e os níveis de realização académica apropriados à escolaridade. A importância das habilidades sociais é reforçada por Michelson, Sugai, Wood e Kazdin (1987), ao dar conta do seu impacto ao nível da adaptação social, emocional e académica da criança. Aqui, destacamos a importância da assertividade, a qual inclui a capacidade adequada de auto-afirmação, auto-expressão e defesa de opiniões, necessidades, sentimentos e insatisfações próprias, sem violar os direitos dos outros (Casares, 2009; Jardim \& Pereira, 2006; Torrego \& Moreno, 2003). Por seu turno, o ajustamento comportamental é manifesto através de problemas de internalização (por exemplo, timidez, isolamento, aansiedade) e/ou externalização (hostilidade, agressividade e antissocialidade) (Lemos, 1997).

\section{A interligação entre os construtos CE e CS}

Atendendo à interligação entre os construtos de CS e CE (Denham, 2007; McCabe \& Altamura, 2011), vários autores (e.g. Ashiabi, 2007 cit. in McCabe \& Altamura, 2011; Denham et al., 2003) referem-se a um único construto de competência sócio-emocional, cujos indicadores incluem aspetos como interações positivas com os educadores/professores e com os pares, representações positivas do self formadas a partir de relações de vinculação positivas, compreensão das emoções, competências de regulação emocional, competências de resolução de problemas, e aceitação pelos pares (Denham et al., 2003), considerando-se que a competência emocional das crianças promove a sua crescente competência social e vice-versa (Denham, 2007). Uma vez que as crianças com problemas comportamentais tendem a apresentar défices ao nível das competências emocionais e sociais e que a combinação de tais problemas as colocam em risco considerável para o desenvolvimento de psicopatologia (Domitrovich, Cortes, \& Greenberg, 2007).

\section{Promoção das competências sócio-emocionais}

Teixeira (2010), refere que desenvolvimento de competências emocionais e sociais surge como um processo contínuo e constante, funcionando de forma complementar ao desenvolvimento cognitivo, visando o desenvolvimento da inteligência emocional e social e da capacidade do indivíduo se desenvolver emocionalmente de forma positiva e eficaz.

\section{O recurso ao jogo como ferramenta de promoção de competências emocionais e sociais}

A discussão sobre o desenvolvimento infantil através do jogo é expressa por teóricos como Piaget (1978) e Vygotsky (1991). Segundo Piaget (1978), ao jogar, a criança assimila a realidade e, desta forma, transforma-a em função das suas necessidades. A utilização do jogo como recurso para a promoção de comportamentos pode revelar-se uma ferramenta útil de apoio ao trabalho psicoterapêutico. O jogo representa, por exemplo, uma 
oportunidade para a aplicação de estratégias comportamentais como sejam, a modelagem, o feedback positivo e os ensaios comportamentais. Atendendo a que as crianças com défices sócio-emocionais podem apresentar prejuízos nas interações sociais, a situação de jogo proporciona o acesso a sentimentos e a outros comportamentos encobertos, na medida em que, ao jogar, a criança expressa o que sente e o que pensa (Macedo, Pitty \& Passos, 2000; cit. Tintori, Bast \& Pitta, 2010). A condução do jogo pelo terapeuta favorece a observação e análise dos comportamentos da criança e permite direcionar os procedimentos à gestão das contingências envolvidas. Através do jogo, diante de um quadro de baixas competências sociais, podemos promover aquisições tais como: o desenvolvimento do autocontrolo, a melhoria da auto-estima e das relações interpessoais, estratégias mais adequadas para lidar com os sentimentos negativos, e a tomada de decisão.

Do ponto de vista da relação terapêutica, o contacto da criança com o jogo facilita o desenvolvimento de uma boa relação entre a díade terapêutica, contribuindo para que capacidades motoras, cognitivas, sociais e académicas, muitas vezes deficitárias, sejam desenvolvidas mediante um ambiente reforçador (Shives, 2007 cit. Tintori, Bast \& Pitta, 2010).

\section{Apresentação do Jogo "Agora Tu"}

O jogo de tabuleiro "Agora Tu" tem como principal motivação a procura de um instrumento de trabalho que possa favorecer a aquisição de comportamentos sócioemocionais. Além disso, reflete a importância das atividades lúdicas, amplamente reconhecidas pela literatura, no incitamento das crianças a tornarem-se participantes mais ativos - quer física quer verbalmente (Martyniuk \& Dworkin, 2011; Sarno \& Wurtele, 1997) - no seu próprio processo de aprendizagem.

\section{Objetivos do Jogo "Agora Tu”}

O jogo foi criado para ser usado em contexto terapêutico, com crianças dos 7 aos 10 anos de idade. Estando ainda em fase de teste, o jogo encontra-se em contínuo processo de atualização. Este instrumento visa atuar não só como estratégia preventiva do desajustamento pessoal, escolar e social da criança, mas também como estratégia otimizadora da sua saúde e bem-estar, trabalhando, de acordo com a literatura, a promoção: (i) de competências de identificação, regulação e expressão de emoções; (ii) do autoconhecimento e de competências de valorização pessoal; (iii) de estilos de comunicação adequados; (iv) da capacidade de tomada de decisão responsável e construtiva; (v) da aplicação de procedimentos comportamentais, como reforço de comportamentos adequados, extinção de inadequados e modelagem, e ainda (vi) da consciência social e resolução de problemas interpessoais (e.g., Lopes et al., 2006; Webster-Stratton, 2012).

\section{Descrição da estrutura do Jogo}

Em si, o jogo é composto por um tabuleiro, onde estão representados dois contextos (familiar e escolar), divididos em diferentes casas, associadas a um determinado desafio-tema. O jogo apresenta cinco módulos que abordam temas encontrados na literatura como sendo relevantes para a promoção das diferentes competências sócio-emocionais (e.g., Denham, 2007; McCabe \& Altamura, 2011; Webster-Stratton, 2012). A nível estrutural, o jogo define-se pelos seguintes temas: (1) Compreender as Emoções; (2) Autoconhecimento e Auto-estima; (3) Estilos de Comunicação; (4) Tomada de Decisão; (5) Consciência Social e Resolução de Problemas Interpessoais.

\section{Aplicação do Jogo}

O jogo é utilizado durante 10 semanas, com, com a duração de 50 minutos por sessão.

\section{Método}

A seleção dos participantes foi feita através de um processo de amostragem não probabilístico, de conveniência (Johnson \& Christensen, 2007). O estudo pré-teste envolveu a aplicação junto de três crianças com idades compreendidas entre os 9 e os 10 anos de idade. Participaram ainda, cinco psicólogos com experiência no trabalho com crianças e adolescentes. Os psicólogos estavam divididos entre dois aplicadores e três juízes. Os dados recolhidos da avaliação dos juízes foram analisados com o recurso à análise de conteúdo categorial (Bardin, 2009).

\section{Participantes}

Crianças: Três crianças de 9 e 10 anos que se encontravam em acompanhamento psicológico. As crianças frequentavam o $4^{\circ}$ e o $5^{\circ}$ ano do primeiro e segundo ciclo do ensino básico, respectivamente.

Psicólogos: Os dois psicólogos que aplicaram o jogo têm experiência profissional em terapia cognitivocomportamental. As suas idades são 35 e 45 anos.

Juízes: Os três juízes que avaliaram o conteúdo do jogo têm formação superior em Psicologia, com experiência profissional em terapia cognitivocomportamental. Eram do sexo feminino, com idades compreendidas entre os 30 e os 40 anos.

\section{Instrumentos}

Pais e Crianças: Ficha sociodemográfica para registo de dados dos pais e dados relativos à criança. Entrevista clínica junto dos pais sobre a criança. No final de cada sessão era realizada uma reflexão com a criança sobre os conteúdos da sessão e sobre o que aprendeu. Avaliação de pré-teste e pós-teste: Questionário de Comportamentos da Criança - CBCL (Achenbach \& Rescorla, 2001), preenchidos pelos pais das crianças.

Juízes: Foi elaborada um grelha de análise das questões e do manual que compõem o jogo. No número de questões para orientação do processo de análise dos juízes incluíam-se: (1) o grau de adequação do conteúdo, (2) a pertinência, (3) a relevância, e (4) o grau de diversidade dos conteúdos.

\section{Procedimento}

A experimentação aos materiais que compõem o jogo foi dividida em oito fases: i) Foi elaborado o programa de competências sócio-emocionais de acordo com a 
literatura mais recente; ii) foi feita a elaboração das atividades que foram colocadas em cartas; iii) Foram criadas as instruções, o manual e o tabuleiro do jogo; iv) Foi determinado o número de atividades necessárias para chegar ao fim do jogo; v) Foi realizada a avaliação do conteúdo das cartas com o objetivo de confirmar se o jogo tratava aquilo a que ele se propunha tratar. Para o efeito, o jogo foi apresentado a três juízes. vi) Foi estabelecida uma sequência de apresentação das cartas; vii) Relativamente à aplicação, seguiu-se a explicação do jogo e seus objetivos aos pais, agradecendo a sua autorização; viii) Deu-se início à aplicação do jogo com três crianças. O jogo está a ser submetido à apreciação da comunidade científica num evento internacional. Isto permitir-nos-á perceber a pertinência do desenvolvimento e estandardização do instrumento, fazendo os devidos ajustes para uma sua melhor otimização.

\section{Resultados da aplicação experimental}

\section{Resultados da avaliação dos juízes}

As categorias surgiram dos dados e destes emergiram subcategorias. As seis grandes categorias foram: Adequado ao objetivo; Relevância; Vantagem; Linguagem e compreensão; Atividades; Sugestões de atividades. São exemplos de subcategorias: Conscientização das consequências; Aquisição de comportamentos sociais e emocionais; Melhorar as interações sociais. A Figura 1. apresenta as categorias relativas aos resultados da avaliação dos juízes. As apreciações realizadas pelos juízes demonstraram que a técnica desenvolvida estava a ser coerente com a base teórica proposta, por exemplo, "Todas as atividades que fazem parte integrante deste jogo, vão ao encontro dos conceitos envolvidos ao nível das competências sócioemocionais". De uma forma geral, o jogo foi apreciado pelos Juízes: "Penso que o jogo foi bem pensado e estruturado". Na categoria Adequado ao objetivo, os juízes assinalaram que o jogo é capaz de promover competências sócio-emocionais, fomentando ainda a consciencialização das consequências dos comportamentos nas crianças. Quanto à categoria Relevância, os juízes são unânimes a referir que o jogo "faz todo sentido" e "A utilização deste jogo pode-se mostrar vantajosa, no sentido de antever possíveis dificuldades ao nível sócio-emocional". As vantagens enumeradas são diversificadas e remetem para a subcategoria "Aquisição de comportamentos sociais e emocionais" que se subdivide em: estimular a reflexão e o autoconhecimento; promover a importância da comunicação e expressão dos sentimentos, pensamentos e comportamentos; aprender comportamentos alternativos e funcionais; melhorar as interações sociais; e promover a saúde mental. Além disso, o jogo foi apontado como uma possibilidade de antever possíveis dificuldades ao nível sócio-emocional, precavendo possíveis problemas de comportamento. A linguagem do jogo e sua compreensão suscitou opiniões diferentes, sendo que um juiz considerou que a linguagem não é adequada à faixa etária a que se propõe o jogo. De igual forma, apenas um juiz considerou que as regras não eram de fácil compreensão, requerendo ajustes.

Quanto às tarefas que compõem o jogo, os três juízes consideraram na sua maioria, pertinentes, criativas, motivantes. Uma chamada de atenção foi realizada para a passagem de nível, dada a necessidade de conclusão correta da tarefa anterior, facto que foi considerado desmotivador por um juiz. No que respeita a categoria Sugestões, foram apresentadas pelos juízes a sugestão de acrescentar um tópico sobre a relação com o adulto (pais, familiares, professores).

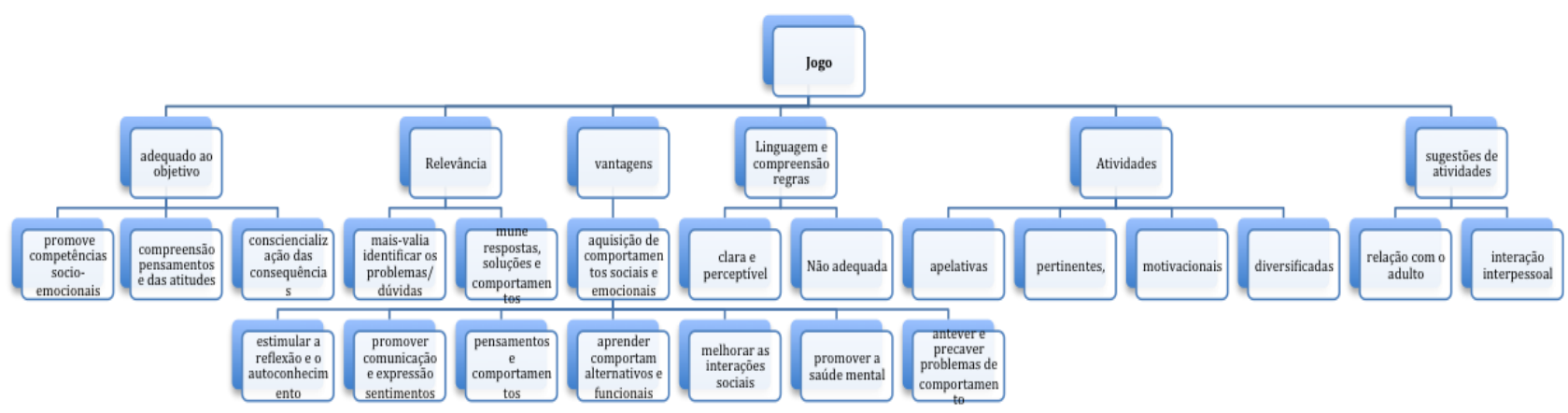

Figura 1. Categorias relativas aos resultados da avaliação dos juízes

\section{Resultados da aplicação pelos psicólogos}

A avaliação dos psicólogos aplicadores apresenta-se como predominantemente positiva. Num primeiro momento destaca-se a motivação das crianças para a realização do jogo, seguida da conclusão da sua adequação e relevância para aquilo a que se propõe, ou seja, aquisição de comportamentos e atitudes sociais e emocionais adequadas. Os psicólogos consideraram que o jogo em análise é um instrumento promotor da 
comunicação dos sentimentos, do autoconhecimento e da identidade, bem como da capacidade de tomar decisões de forma responsável e construtiva e da regulação emocional.

\section{Resultados da aplicação experimental com as crianças}

A aplicação experimental do jogo ocorreu entre outubro de 2016 e março de 2017. As crianças tiveram uma atitude positiva em relação ao jogo, sendo este percebido como relevante e original por duas das três crianças. Todas as crianças $(\mathrm{N}=3)$ demonstraram compreender as instruções do jogo e repetir por palavras suas o que era pedido. As atividades que demonstraram mais dificuldade foram aquelas que envolviam temas mais teóricos.

\section{Discussão}

Os resultados alcançados com esta iniciativa experimental permitem-nos afirmar que o jogo apresentado promove nas crianças o conhecimento sobre temas que a literatura sugere deverem ser abordados no âmbito da otimização de competências sócio-emocionais. Baseando a primeira versão do jogo na literatura relevante acerca destes conteúdos nomeadamente, diversos programas já existentes (e.g., Denham, 2007; Lopes et al., 2011; Saarni, 1999) -, a realização do pré-teste permitiu-nos analisar em que medida este jogo se pode constituir como relevante para o robustecimento e diversificação de um repertório de competências sócio-emocionais, e as problemáticas associadas ao seu déficit, avaliando simultaneamente o seu grau de adequação, funcionalidade, e carácter apelativo para população a que se destina.

$\mathrm{O}$ envolvimento dos vários agentes neste processo de experimentação foi de suma importância para a construção de uma ferramenta adequada ao trabalho psicoterapêutico. Atendendo aos resultados da avaliação dos juízes e dos psicólogos que aplicaram o jogo, podemos afirmar que o mesmo está adequado à faixa etária a que se propõe, sendo bastante relevante pelos temas que aborda e, simultaneamente, um instrumento importante para a promoção de competências sócioemocionais. A única ressalva feita refere-se à linguagem utilizada, sendo sugerida uma maior adequação à idade da população-alvo do jogo, aspecto que será acautelado na próxima aplicação, tendo ainda sido apresentadas algumas sugestões de acréscimos ao teor das atividades, o que será igualmente considerado no futuro. Considerase uma mais-valia o facto de o jogo ter sido experimentado integralmente por crianças na faixa etária do público-alvo e por técnicos da área. Como limitações apontamos a dimensão da amostra. O jogo, inédito por constituir uma intervenção psicoeducativa rara em Portugal, permite colmatar a necessidade sentida pelos psicólogos portugueses ao nível da promoção de competências sócio-emocionais em crianças através do jogo, oferecendo um instrumento de trabalho propiciador da eficácia na promoção do sucesso escolar, na gestão de conflitos sócio-familiares e na resistência emocional a situações problemáticas, bem como no ajustamento psicológico.

\section{Referências}

Abe, A. \& Izard, C. (1999). A longitudinal study of emotion expression and personality relations in early development. Journal of Personality and Social Psychology, 77(3), 566-577.

Asher, S. (1990). Recent advances in the study of peer rejection. In S.R. Asher \& John D. Coie (Eds.). Peer rejection in childhood (pp.3-16). New York: Cambridge studies.

Bardin, L. (2009). Análise de conteúdo (Edição revista e actualizada). Lisboa: Edições, 70.

Belacchi, C. \& Farina, E. (2010). Prosocial/hostile roles and emotion comprehension in preschoolers. Aggressive Behavior, 36, 6, 371-389.

Bohnert, A. M., Crnic, K. A., \& Lim, K. G. (2003). Emotional competence and aggressive behavior in school-age children.Journal of Abnormal Child Psychology, 31(1), 79-91.

Bosacki, S. \& Moore, C. (2004). Preschoolers' Understanding of Simple and Complex Emotions: Links with Gender and Language. Sex Roles, 50, 910, 659-675.

Bruner, J. (1978). O processo de educação. São Paulo: Nacional.

Casares, M. I. (2009). Cómo promover la convivencia: Programa de asertividad y hablidades sociales (PAHS) (2 $2^{\mathrm{a}}$ ed.). Madrid: Narcea.

Demaray, M. K., \& Elliott, S. M. (2001). Perceived social support by children with characteristics of ADHD. School Psychology Quarterly, 16, 68-90.

Denham, S. A. (2007). Dealing with feelings: How children negotiate the worlds of emotions and social relationships. Cognition, Brain \& Behavior, 11, 1-48.

Denham, S., Blair, K., DeMulder, E., Levitas, J., Sawyer, K., Auerback-Major, et al. (2003). Preschool emotional competence: Pathway to social competence? Child Development, 74, 238-256.

Dennis, T. A., Cole, P. M., Zahn-Waxler, C., \& Mizuta, I. (2002). Self in context: Autonomy and relatedness in Japanese and U.S. mother-preschooler dyads. Child Development, 73, 1803-1817.

Domitrovich, C. E., Cortes, R. C., \& Greenberg, M. T. (2007). Improving young children's social and emotional competence: A randomized trial of the preschool "PATHS" curriculum. The Journal of Primary Prevention, 28, 2, 67-91.

Figueiredo, N. (2015). Vamos Prevenir! do Búzio e da Coral - Jogo de Prevenção Primária do Abuso Sexual para Crianças entre os 6 e os 10 anos. Tese de Mestrado não publicada, ISCTE-IUL.

Gross, J. (1998). The emerging field of emotion regulation: An integrative review. Review of General Psychology, 2, 271-299.

Huizinga, J. (2007). Homo ludens: o jogo como elemento da cultura ( $5^{\text {a }}$.Ed). São Paulo: Perspectiva.

Lazarus, R.S. (2006). Emotions and interpersonal relationships: Toward a person centered 
conceptualization of emotions and coping. Journal of Personality, 74, 9-46.

Lopes, J., Rutherford, R., Cruz, M. C., Mathur, S., \& Quinn, M. (2011). Competências sociais: Aspectos comportamentais, emocionais e da aprendizagem ( $2^{\mathrm{a}}$ ed.). Braga: Psiquilibrios.

Lourenço, S., Palmeira, L., Dinis, A., \& Pinto-Gouveia, J. (2010). Validação das emoções na infância: vergonha, ansiedade e sintomatologia depressiva. Psychologica, 52, 2, 499-526.

McCabe, P., \& Altamura, M. (2011). Empirically valid strategies to improve social and emotional competence of preschool children. Psychology in the Schools. 48, 5, 513-540.

Melo, A. I. (2005). Emoções no período escolar: estratégias parentais face à expressão emocional e sintomas de internalização e externalização da criança. Dissertação de Mestrado, Universidade do Minho, Minho

Michelson, L., Sugai, D. P., Wood, R. P., \& Kazdin, A. E. (1987). Lãs habilidades sociales en Ia Infância. Barcelona. Martínez Roca.

Piaget, J. (1978). A formação do símbolo na criança: imitação, jogo e sonho, imagem e representação. Rio de janeiro: Guanabara-Koogan.

Pons, F., Harris, P. L., \& Rosnay, M. (2004). Emotion comprehension between 3 and 11 years: Developmental periods and hierarchical organization. European Journal of Developmental Psychology, 1, 127-151.

Rubin, K. D., \& Daniels-Byrness, T. (1983). Concurrent and predictive correlates of sociometric status inkindergarten and grade 1 children. Merrill-Palmer Quarterly, 29, 337-352.

Saarni, C. (1999). The development of emotional competence. New York: Guilford Press.

Salovey, P., \& Mayer, J. D. (1990). Emotional intelligence. Imagination, Cognition and Personality, 9, 3, 185-221.

Sarno, J. A., \& Wurtele, S. K. (1997) Effects of a personal safety program on preschoolers' knowledge, skills, and perceptions of child sexual abuse. Child Maltreatment, 2, 35-45.

Tintori, F., Bast, D. \& Pitta, M. (2013). Jogo na terapia comportamental em grupo de crianças com TDAH. Acta comportamentalia, 19, 2, 225-239, 2011.

Teixeira, S. (2010). Jogos, brinquedos, brincadeiras e brinquedoteca: Implicações no processo de aprendizagem e desenvolvimento. Rio de Janeiro: Wak Editora.

Torrego, J. C., \& Moreno, J. M. (2007). Convivencia y disciplina en la escuela. El aprendizaje de la democracia. Madrid: Alianza Editorial.

Vygotsky, L. (1991). A formação social da mente. São Paulo: Martins Fontes.

Webster-Stratton, C. (2012). Os anos incríveis - Guia de resolução de problemas para pais de crianças dos 2 aos 8 anos de idade. Braga: Psiquilíbrios Edições. 